\title{
Rettungsdienstliche Strategie beim Hängetrauma
}

Raimund Lechner, Enrico Staps, Hermann Brugger, Simon Rauch

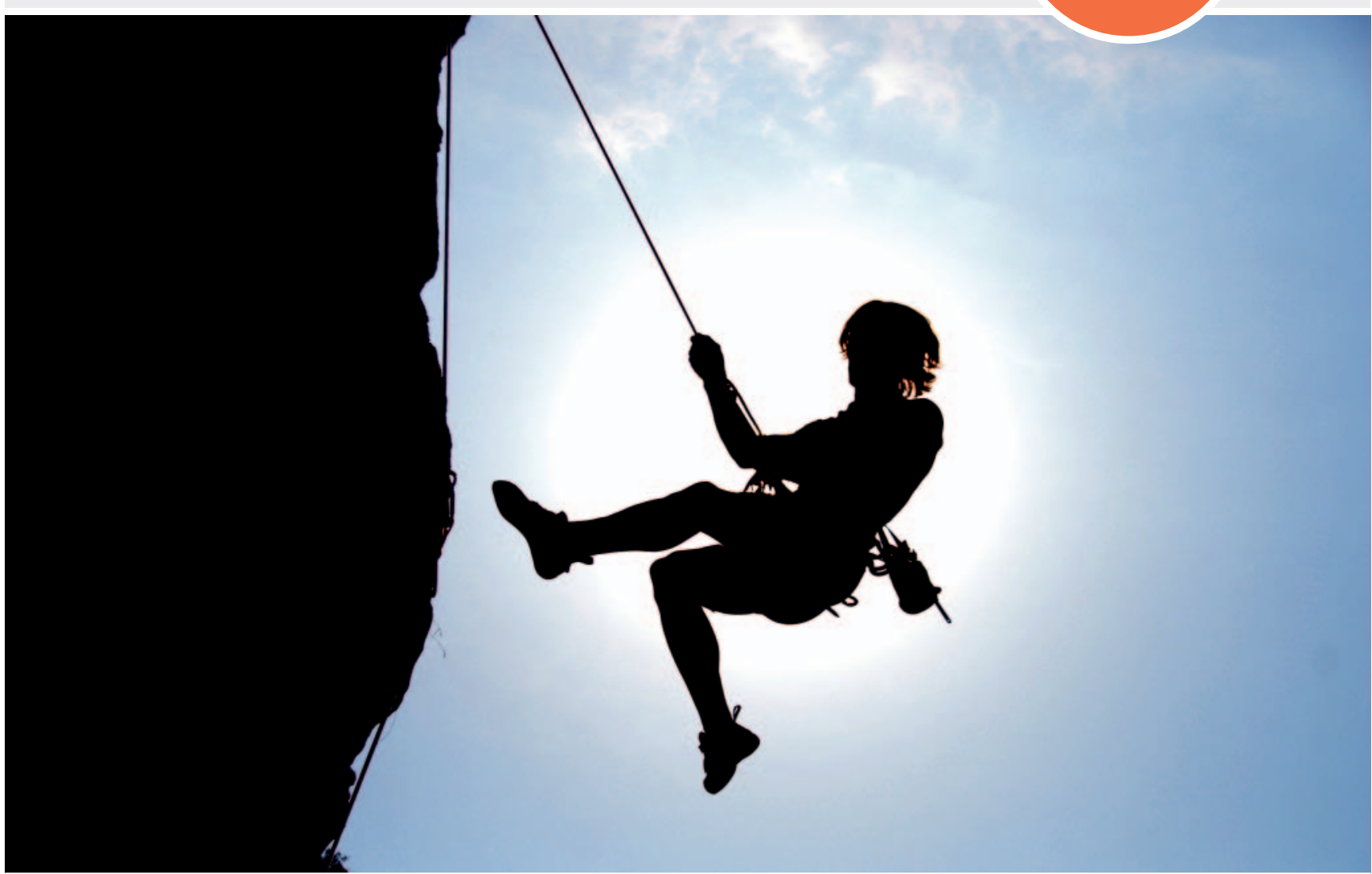

Quelle: Rémy MASSEGLIA/AdobeStock.com

Das Hängetrauma beschreibt eine durch bewegungsloses, annähernd vertikales freies Hängen in einem Anseilgurt induzierte Kreislaufdepression mit möglicher Todesfolge. Hauptsächlich betroffene Personengruppen sind somit Kletterer, Bergsteiger und seilgesicherte Arbeiter. Die zügige Rettung des Patienten unter Beachtung des Eigenschutzes ist von herausragender Bedeutung. Die Akutbehandlung nach einem Hängetrauma folgt dem Standard-<c>ABCDE-Algorithmus mit initialer Flachlagerung.

\section{Einleitung}

\section{DEFINITION}

\section{Hängetrauma}

Das Hängetrauma beschreibt ein potenziell lebensbedrohliches Ereignis, das durch freies passives Hängen in nahezu vertikaler Position eintritt [1].

Ursächlich für das Versterben ist ein im Wesentlichen durch eine generalisierte Hypoperfusion induziertes Multi- organversagen. Da es sich dabei nicht um eine Verletzung im eigentlichen Sinne handelt, werden im internationalen Sprachgebrauch mittlerweile statt des ursprünglich eingeführten Begriffs Hängetrauma („suspension trauma“) oft die Begriffe „suspension syndrome“, „harness hang syncope“ oder ähnliche Begriffe verwendet, die die Kausalkette begrifflich besser erfassen [2, 3].

Das Hängetrauma spielt in der Arbeitswelt eine Rolle, wenn Arbeiten seilgesichert durchgeführt werden. 
Zudem kommt es im Rahmen von seilgesicherten Freizeitaktivitäten vor, wie z. B.

- (Eis)Klettern,

- Bergsteigen,

- Canyoning,

- Speläologie (Höhlenkunde),

- Fallschirmspringen,

- Paragliden.

1972 wurde die erste Fallserie auf der 2. Internationalen Bergrettungsärztetagung in Innsbruck präsentiert [4]. Eindeutige Aussagen zur Inzidenz sind nicht möglich, es existieren jedoch zahlreiche Fallberichte [1, 3, 5-7]. Zudem konnten sowohl in Hängeversuchen als auch in Kipptischversuchen Kreislaufveränderungen und Präsynkopen induziert werden, wobei die minimale Zeitdauer bis zum Eintritt einer Präsynkope teilweise lediglich wenige Minuten betrug [2, 8, 9].

\section{Pathophysiologie}

Seit der Erstbeschreibung wurden verschiedenste pathophysiologische Kausalketten diskutiert [10]. Vor allem 2 Mechanismen scheinen bei der Entwicklung eines Hängetraumas eine relevante Rolle zu spielen. Zum einen kommt es schwerkraftbedingt zu einem venösen Blutpooling in den abhängenden Körperpartien, vor allem in den Beinen. Verstärkt wird dies durch eine Reduktion der Muskelpumpe infolge einer verminderten Muskelaktivität, beispielsweise durch

- Erschöpfung,

- Hypoglykämie,

- Schmerzen,

- Verletzungen,

- Hypothermie und

- Bewusstlosigkeit aus anderen Gründen.

Dies führt zu einer Reduktion der kardialen Vorlast und konsekutiv zu einem reduzierten Herzminutenvolumen mit einer generalisierten Hypoperfusion $[1,3,10]$.

Zudem scheint eine vasovagale Reflexantwort eine Rolle zu spielen [1, 3]. So wird postuliert, dass der reduzierte venöse kardiale Rückstrom über den Bezold-JarischReflex eine Bradykardie mit konsekutiver Bewusstlosigkeit auslösen kann, ähnlich einer vasovagalen Synkope [3, 11]. Die pathophysiologische Grundlage einer Synkope ist die vorübergehende zerebrale Unterversorgung mit Blut und somit Sauerstoff. Eine verminderte zerebrale Sauerstoffsättigung konnte bei in Hängeversuchen synkopierten Probanden nachgewiesen werden [2].

Bei einem durch Tonusverlust bedingten synkopalen Sturz kommt es gewöhnlich zu einer horizontalen Position des Patienten und damit zur Wiederherstellung einer adäquaten zerebralen Perfusion, verbunden mit einem

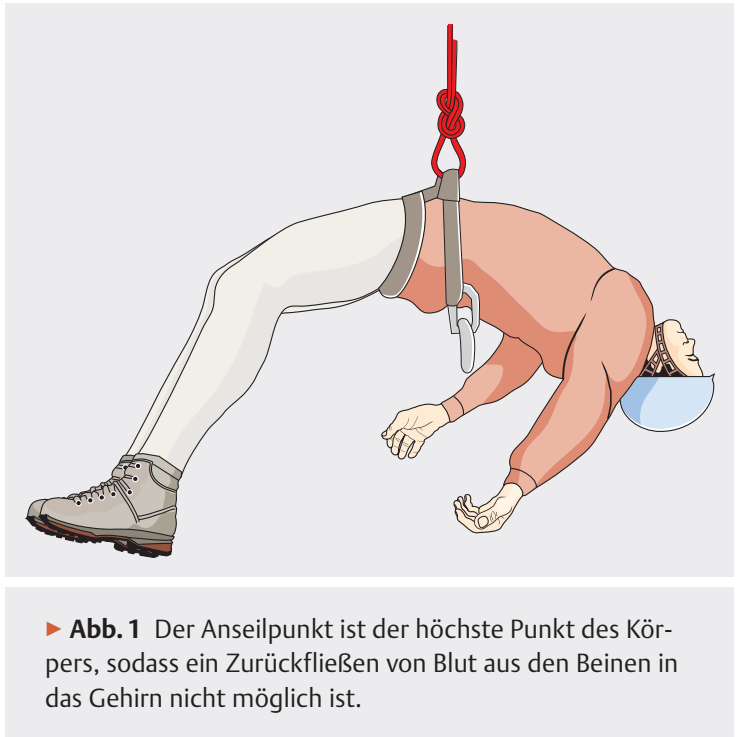

zügigen Aufklaren des Patienten [12, 13]. Beim an einem Seil fixierten Patienten stellt hingegen der Anseilpunkt den höchsten Punkt dar ( $\triangleright$ Abb. 1 u. [3]). Ein Rückfluss von Blut aus den Beinen ist somit nicht möglich. Die Minderperfusion wird nicht vollumfänglich aufgehoben, was bei ausreichend langer Hängedauer zum Versterben des Patienten führen kann.

Merke

Ein venöses Pooling und eine vasovagale Reflexantwort scheinen eine tragende Rolle in der Pathophysiologie des Hängetraumas zu spielen. Eine zerebrale Reperfusion mit zügigem Aufklaren nach Tonusverlust bleibt aufgrund der hängenden Position des Patienten allerdings aus.

Obwohl die vasovagale Reflexantwort bei allen gesunden Individuen vorhanden ist, scheinen individuelle Unterschiede bezüglich der Anfälligkeit zu bestehen [14]. Dementsprechend kann die Zeit bis zum Symptombeginn und vom Symptombeginn bis zum Eintritt der Bewusstlosigkeit interindividuell enorm variieren, nicht valide vorhergesagt werden und im Extremfall nur einige Minuten betragen [1, 3, 9].

Bei einem am Seil fixierten Bewusstlosen kann es zusätzlich durch eine schwerkraftbedingte Hyperflexion oder Hyperextension des Halses zu einer Verlegung des Atemwegs mit möglicher Todesfolge kommen [5, 6]. Darüber hinaus führt die allgemeine Gewebehypoperfusion und damit Gewebehypoxie zu einer Zellschädigung, vor allem im Muskelgewebe.

Außerdem können möglicherweise eine Blutstase, verursacht durch den verminderten Blutrückfluss aus den Beinen, sicher jedoch eine Hypothermie als Folge des langen bewegungslosen freien Hängens und eine exzessive 


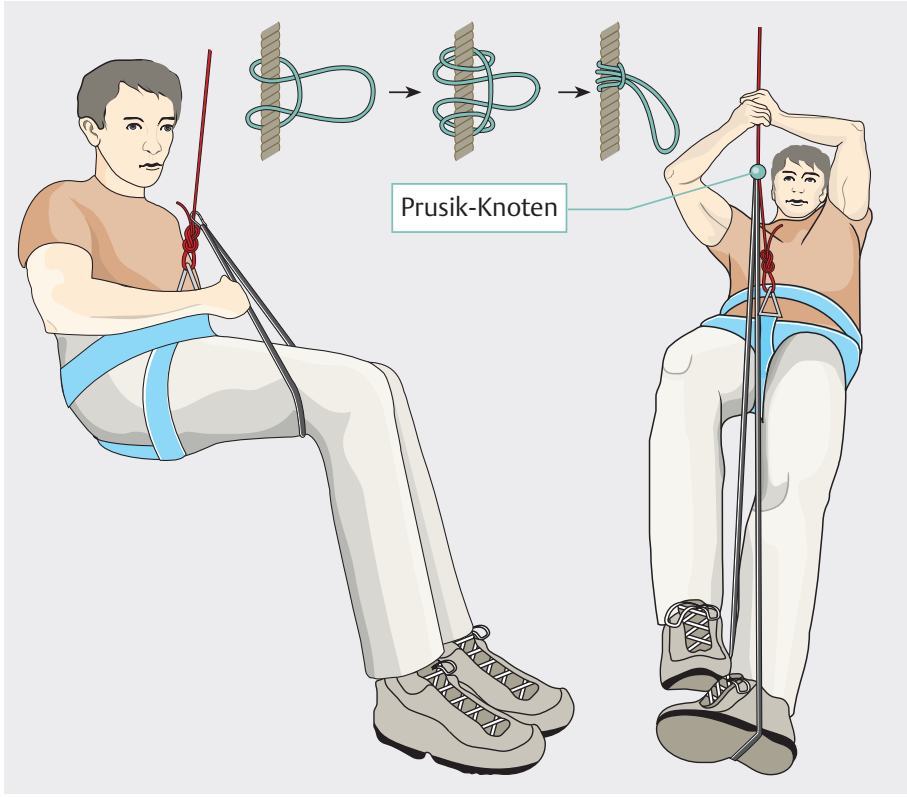

Abb. 2 Trittschlingen, die mithilfe von Klemmknoten (z. B. Prusik-Knoten [s. Abbildung], Kreuzklemmknoten) oder auch Klemmgeräten am Seil befestigt werden können, um durch wiederholtes Hineintreten die Muskelpumpe und damit den venösen Blutfluss zu aktivieren. Zusätzlich kann durch eine Schlinge unter den Kniekehlen die Lagerung hin zu einer horizontalen Position optimiert werden.

Muskelarbeit aufgrund protrahierter Selbstrettungsversuche zu einer Muskelzellnekrose führen [15-17].

Eine durch die multifaktorielle Rhabdomyolyse verursachte Hyperkaliämie kann lebensbedrohliche kardiale Arrhythmien verursachen. Außerdem können die mit der Rhabdomyolyse verbundene Myoglobinämie und die Minderperfusion der Nieren bei langen Hängezeiten eine akute Nierenschädigung verursachen (prä- und intrarenales Nierenversagen - Crush-Niere) [3, 6, 7].

Bisher konnte wissenschaftlich nicht eindeutig geklärt werden, ob in erster Linie das venöse Pooling in den unteren Extremitäten oder ein vasovagaler Reflex für das Kreislaufversagen beim freien Hängen im Seil verantwortlich ist. Aus der vorliegenden Literatur kann man jedoch schließen, dass beide Faktoren eine wesentliche Rolle spielen. Weitere Untersuchungen sind nötig, um vor allem den zeitlichen Verlauf der Kreislaufparameter während der Hängephase zu klären.

\section{Prävention}

Aufgrund der potenziellen Lebensgefahr durch freies Hängen im Seil muss sich jede gefährdete Person zwingend des Hängetraumas bewusst sein. Unterrichtung und Ausbildung sind die Basis jedweder Präventionsstrategie.

\section{Selbstrettung/Kameradenrettung}

Da die Inzidenz unklar ist und auch der Eintritt einer Bewusstlosigkeit interindividuell sehr verschieden ist, sollte die freie Hängezeit prinzipiell so kurz wie möglich gehalten werden. Dies impliziert eine möglichst schnelle Selbst- oder Fremdrettung.

\section{Merke}

Eine schnelle (Selbst-)Rettung ist von höchster Bedeutung.

Voraussetzung dafür ist, dass die hängende Person über Selbstrettungsfertigkeiten verfügt und auch das dafür notwendige Material mit sich führt. Dies umfasst prinzipiell Aufstiegstechniken am Seil sowie Abseiltechniken. Bezüglich Detailinformationen wird auf einschlägige Lehrbücher, beispielsweise der Alpenvereine, verwiesen [18 - 20].

Sollte die hängende Person nicht in der Lage sein, sich selbst zu retten, sei es wegen Erschöpfung, Verletzungen, Hypoglykämie, Hypothermie, Schmerzen, präsynkopaler Symptome oder Bewusstlosigkeit, muss es Ziel der Begleitpersonen sein, die hängende Person so schnell wie möglich an einen Ort zu verbringen, an dem die Person flach gelagert werden kann [1, 6].

\section{Merke}

Ähnlich wie bei der Lawinenrettung ist die Selbst- und Kameradenhilfe das erste und mit Abstand wichtigste Glied in der Überlebenskette und hinsichtlich ihres Einflusses auf die Prognose deutlich höher einzuschätzen als die organisierte professionelle Rettungskette. Seilgesicherte Tätigkeiten sollten deshalb niemals allein ausgeführt werden [1, 2].

Da ein venöses Pooling in der Pathophysiologie eine tragende Rolle zu spielen scheint, werden bei zeitlich verzögerten Rettungen Maßnahmen empfohlen, die den venösen Rückfluss aus den abhängenden Körperteilen stimulieren. Dies kann hauptsächlich durch die Aktivierung der Muskelpumpe erfolgen, beispielsweise durch forcierte Beinbewegungen („Luftradfahren“) oder durch Abstoßen von einer in Reichweite befindlichen Haus-, Fels-, Gletscherspaltenwand oder Ähnlichem [1, 3, 6, 21].

Falls keine solchen Strukturen in Reichweite sind, können mit Klemmknoten befestigte Schlingen oder Trittleitern als Widerlager verwendet werden $[1,3,6]$. Auch eine mehrfache Umwicklung des Fußes mit einem eventuell vorhandenen freien Seilstück kann als Widerlager für den Fuß dienen.

Zusätzlich können die Beine durch Anbringen einer möglichst breiten Schlinge unterhalb der Knie in eine annähernd horizontale Position gebracht werden, was den venösen Blutrückfluss erleichtert ( $\triangleright$ Abb. 2) $[3,5,8]$. 
Um das freie Hängen an sich erträglicher zu machen, kann zusätzlich eine Schlinge unter den Achseln durchgeführt und im Anseilpunkt fixiert werden. Dies entlastet die Oberkörpermuskulatur und die Atemhilfsmuskulatur beim freien Hängen in einem Hüftgurt [6].

\section{PRAXIS}

Tipp

Eine Aktivierung der Muskelpumpe während des freien Hängens reduziert das Risiko, ein Hängetrauma zu erleiden.

\section{Ausrüstung}

Unter präventiven Gesichtspunkten ist bei der Wahl des Anseilgurts Folgendes zu beachten:

Die Nutzung von Sicherungsgürteln, die alleinige Verwendung von Brustgurten (ohne Sitzgurt) sowie das direkte Einbinden mit dem Seil um den Körper sind aufgrund des äußerst schmerzhaften freien Hängens und des Eintretens einer kritischen Kompressionsasphyxie obsolet $[1,3,9]$.

Gurte mit einem hohen sternalen Einbindepunkt und Auffanggurte mit am Rücken befindlichem Anseilpunkt (oft im Arbeitsschutz als Sicherung gegen Absturz verwendet, da sie ein freies Bewegen der Arme vor dem Körper ermöglichen) müssen kritisch betrachtet werden, wenn es zu einem freien Hängen kommen kann ( $\triangleright$ Abb. 3).

- Erstens kommt es in diesen Gurten zu einer deutlich vertikaleren Hängeposition als bei der Verwendung eines Hüftgurts oder Brust-Sitzgurts. Es ist anzunehmen, dass das venöse Pooling dadurch verstärkt wird.

- Zweitens sind eine Selbstrettung und eine Optimierung der Körperposition während des Hängens durch die ungünstige Seilplatzierung erschwert.

- Drittens ist eine Reduktion des venösen Blutflusses durch direkte Kompression der Femoralgefäße durch das eigene Körpergewicht beim nach vorne gekippten freien Hängen, im Gegensatz zum Hängen mit vor dem Körper liegendem Anseilpunkt, möglich.

- Viertens kommt es bei einer Bewusstlosigkeit und am Rücken liegendem Anseilpunkt schwerkraftbedingt zu einer Flexion des Halses mit einem erhöhten Risiko der Atemwegsverlegung [6].

Generell müssen Gurtsysteme optimal an die Körperproportionen angepasst werden, sodass ein schmerzfreies Hängen möglich ist. Während des passiven freien Hängens soll sich der Körper idealerweise in eine halb horizontale Position einstellen, sodass das venöse Pooling reduziert wird [2, 6, 22]. Bei richtig angepassten Gurten kann eine Gefäßkompression, vor allem der Femoralgefäße, wirkungsvoll vermieden werden [3].

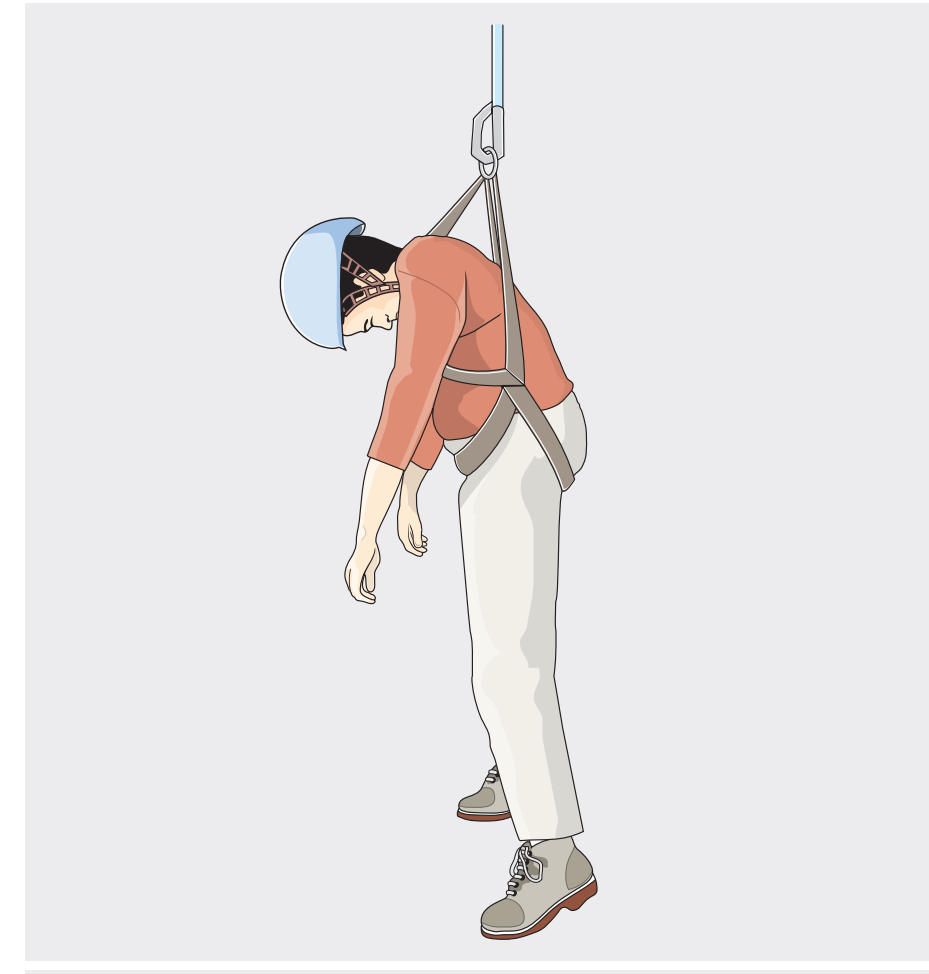

- Abb. 3 Gurtsysteme mit Anseilpunkt am Rücken führen zu einer sehr vertikalen Hänge- und Körperposition und damit zu einer erschwerten Selbstrettung zu einer möglichen Kompression der Femoralgefäße durch das eigene Körpergewicht und - bei Bewusstlosigkeit - zu einer Flexion des Kopfes mit erhöhtem Risiko der Atemwegsverlegung.

\section{PRAXIS}

Tipp

Zusätzlich sollten Rucksäcke vom Rücken genommen und beispielsweise am Anseilpunkt mit einer Schlinge befestigt werden, um lange Hängezeiten besser tolerieren zu können.

\section{Rettungsdienstliches Vorgehen}

Naturgemäß befinden sich Patienten, die ein Hängetrauma erlitten haben, oftmals in absturzgefährdetem Gelände. Der Eigenschutz der Rettungskräfte, vor allem die Absturzsicherung, hat oberste Priorität.

\section{Cave}

Eigenschutz beachten!

Solange der hängende Patient bei Bewusstsein ist, sollten die Rettungskräfte ihn anhalten, die Muskelpumpe, wie oben beschrieben, zu aktivieren. Ist der Patient handlungsunfähig, sollte die erste Person, die den Patienten erreicht, die Beine in eine möglichst horizontale Position anheben. Um ein unbeabsichtigtes Herausrutschen aus dem möglicherweise schlecht angepassten Sicherungs- 
gurt zu vermeiden, kann situationsangepasst der Oberkörper mit einer unter den Achseln durchgeführten und im Anseilpunkt fixierten Schlinge stabilisiert werden [6].

\section{Merke}

Bei bewusstlosen Patienten ist es von allerhöchster Bedeutung, einen freien Atemweg durch Überstrecken des Kopfes zu gewährleisten, vor allem bei Gurten mit einem Anseilpunkt am Rücken.

\section{Lagerung}

Seit der Erstbeschreibung des Hängetraumas gibt es teils emotional geführte Diskussionen bezüglich der initialen Lagerung des Patienten nach der Rettung aus einer hängenden Position. Ursprünglich wurde empfohlen, den Geretteten nicht sofort flach zu lagern, um einen Herzstillstand infolge einer akuten Volumenüberlastung des rechten Ventrikels durch den plötzlichen Rückstrom des Blutes aus der unteren Extremität zu vermeiden [3, 6, 21]. Dies wurde als ursächlich für einen möglichen Bergungstod postuliert, also ein Versterben des Patienten im unmittelbaren zeitlichen Zusammenhang mit der Rettung.

Die Empfehlung, Gerettete vorübergehend in einer Kauerposition zu stabilisieren, kann immer noch in vielen Veröffentlichungen gefunden werden. Aufgrund der Ergebnisse mehrerer Studien kann dies jedoch nicht mehr empfohlen werden. Der überwiegende Teil der aktuellen Literatur sowie die US-amerikanische Bergrettung und das US-amerikanische Pendant zu den Berufsgenossenschaften (Occupational Safety and Health Administration, OSHA) empfehlen folgerichtig eine initiale Flachlagerung mit unverändertem CABCDE-Algorithmus [2, 3, 5, 23-25].

PRAXIS

Prinzip

Die Behandlung nach einem Hängetrauma folgt dem Standard-CABCDE-Algorithmus mit initialer Flachlagerung.

Die Hypothese des Bergungstodes infolge einer akuten Volumenüberlastung des Herzens konnte in wissenschaftlichen Studien niemals nachgewiesen werden. Auch verstarben die meisten Patienten nach Hängetrauma nicht im unmittelbaren zeitlichen Zusammenhang mit der Rettung. Daher kann man in diesen Fällen nicht von einem Bergungstod sprechen. Es handelt sich vielmehr um ein verzögertes Versterben aufgrund anderer pathophysiologischer Vorgänge. Für die wenigen Fallbeschreibungen mit Versterben kurz nach der Rettung sind darüber hinaus alternative Erklärungsansätze wahrscheinlicher. So wird ein Herzstillstand, verursacht durch Hypoperfusion und Hyperkaliämie, als plausibler angesehen [3]. Außerdem erholten sich in zahlreichen experimentellen Studien, in denen ein Hängetrauma künstlich herbeigeführt wurde, alle Probanden nach Flachlagerung, ohne dass es zu einem Herzstillstand gekommen wäre [2, 8, 9, 26].

\section{CABCDE-Algorithmus}

So bald wie möglich sollte also eine Standardbehandlung nach international gängigem CABCDE-Algorithmus erfolgen [3, 5, 21]. Eine EKG-Überwachung ist aufgrund des erhöhten Risikos von Herzrhythmusstörungen so früh wie möglich zu etablieren. Auftretende Herzrhythmusstörungen werden gemäß den aktuell gültigen Leitlinien behandelt [27].

\section{Kardiale Veränderungen}

Aufgrund mehrerer Faktoren sind Patienten mit Hängetrauma einem erhöhten Rhabdomyolyserisiko ausgesetzt [28]. Mit dem erhöhten Risiko einer Rhabdomyolyse geht das Risiko einer Hyperkaliämie und damit von Herzrhythmusstörungen einher. Typische EKG-Zeichen einer Hyperkaliämie sind

- spitze T-Wellen,

- flache oder fehlende P-Wellen,

- breite QRS-Komplexe (>120 ms),

- Sinuswellen (S- und T-Verschmelzungswelle),

- Bradykardie und

- ventrikuläre Tachykardie.

Sollten bei einem Patienten solche EKG-Veränderungen auftreten, kann eine i.v. Kalziumgabe erwogen werden (10 ml Kalziumchlorid $10 \%$ oder $30 \mathrm{ml}$ Kalziumglukonat $10 \%)$. Des Weiteren kann Kalium durch die Vernebelung von 10 - $20 \mathrm{mg}$ Salbutamol nach intrazellulär verschoben werden [29].

\section{Reanimation}

Bei einer Reanimation, bei der ursächlich eine durch ein Hängetrauma induzierte Hyperkaliämie und Azidose angenommen wird, können die Standard-ALS-Reanimationsmaßnahmen um einen schnellen Bolus von $10 \mathrm{ml}$ Kalziumchlorid $10 \%$ i. v. und eine Pufferung mit 50 mmol Natriumbikarbonat erweitert werden [29]. Präklinisch meist nicht verfügbar, aber spätestens innerklinisch kann Kalium durch eine Infusion mit 10 IE kurzwirksames Insulin und $25 \mathrm{~g}$ Glukose nach intrazellulär verschoben werden (Cave: engmaschige Blutzuckerkontrolle) [29].

\section{Flüssigkeits-/Volumenhaushalt}

Bei langen Hängezeiten ist eine liberale kristalloide Flüssigkeitsgabe zur Prävention eines Nierenversagens im Rahmen einer wahrscheinlichen Rhabdomyolyse indiziert [3, 7]. Befand sich der Patient für mehr als 2 Stunden in hängender Position, sollte die Zielklinik über Nierenersatzverfahren verfügen [3]. 


\section{Körpertemperatur}

Da bewegungsloses Hängen das Risiko einer akzidentiellen Hypothermie erhöht, haben Prävention und Behandlung der Hypothermie hohe Priorität [3].

\section{FAZIT}

Die Hauptrisiken bei einem Hängetrauma sind

- während des Hängens:

- Kreislaufdepression und

- Atemwegsverlegung;

- nach der Patientenrettung sind hingegen führend:

- Herzrhythmusstörungen,

- Hypothermie und

- Nierenschädigung.

\section{KERNAUSSAGEN}

\section{Hängetrauma}

Das Hängetrauma ist potenziell lebensbedrohlich!

Eigenschutz!

Prävention

Seilgesicherte Tätigkeiten nicht allein durchführen.

Eine schnelle (Selbst-)Rettung ist von höchster Bedeutung.

Aktivierung der Muskelpumpe (Beinbewegung, Abdrücken gegen Widerlager).

Beim Hängen möglichst horizontale Position, ggf. mit Hilfsschlingen, einnehmen.

Richtige Anpassung der verwendeten Gurtsysteme.

Behandlung

Flachlagerung nach Rettung ist möglich.

Standard ist der CABCDE-Algorithmus.

Frühe Rhythmusüberwachung und Therapie von Herzrhythmusstörungen.

Frühe Diagnose und Therapie einer Hyperkaliämie. Liberale kristalloide Flüssigkeitsgabe.

Ab einer Hängezeit von 2 Stunden Zielklinik mit Nierenersatzverfahren wählen.

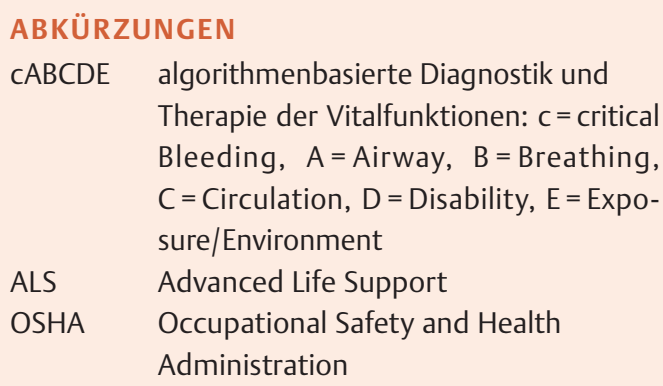

Die Autoren geben an, dass kein Interessenkonflikt besteht.

\section{Autorinnen/Autoren}

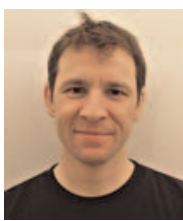

\section{Raimund Lechner}

Dr. med., Oberfeldarzt, Zusatzbezeichnung Notfallmedizin. Tätig als Assistenzarzt in der Klinik für Anästhesiologie, Intensivmedizin, Notfallmedizin und Schmerztherapie am Bundeswehrkrankenhaus in Ulm. Mehrfache Teilnahme am Auslandseinsatz der Bundeswehr in Afghanistan.

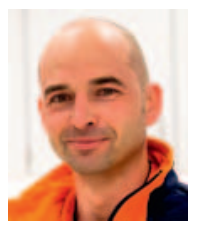

\section{Enrico Staps}

Dr. med., Oberfeldarzt, Facharzt für Anästhesiologie. Zusatzbezeichnungen Intensiv- und Notfallmedizin. Seit 2016 Oberarzt der Klinik für Anästhesiologie, Intensivmedizin, Notfallmedizin und Schmerztherapie am Bundeswehrkrankenhaus Ulm. Notarzt in der Luftrettung und aktives Mitglied der Bergwacht. Schwerpunkte liegen u.a. im Bereich der Berg- und Höhenrettung. Mehrfache Teilnahme an Auslandseinsätzen der Bundeswehr im Kosovo, in Afghanistan und Afrika.

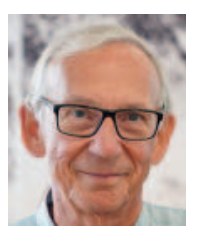

\section{Hermann Brugger}

Prof. Dr. med., Leiter des Instituts für Alpine Notfallmedizin, EURAC research, Bozen, Italien. Medizinische Universität Innsbruck, Präsident der International Society of Mountain Medicine, ISMM.

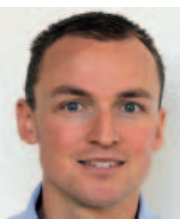

\section{Simon Rauch}

Dr. med., tätig am Institut für Alpine Notfallmedizin, EURAC research, Bozen, Italien, an der Klinik für Anästhesiologie, Klinikum der Universität München, LMU München, sowie am Institut für Sportwissenschaften der Universität Innsbruck.

\section{Korrespondenzadresse}

\section{Oberfeldarzt Dr. med. Raimund Lechner}

Abteilung für Anästhesie und Intensivmedizin

Bundeswehrkrankenhaus Ulm

Oberer Eselsberg 40

89081 Ulm

raimundedgarlechner@bundeswehr.org

\section{Erstveröffentlichung}

Dieser Beitrag ist eine aktualisierte Version des Artikels: Lechner R, Staps E, Brugger $\mathrm{H}$ et al. Notärztliche Strategie beim Hängetrauma. Notarzt 2018; 34: $156-161$. doi:10.1055/a-0632- 3733 . 


\section{Literatur}

[1] Pasquier M, Yersin B, Vallotton L et al. Clinical update: suspension trauma. Wilderness Env Med 2011; 22: 167-171

[2] Lanfranconi F, Pollastri L, Corna G et al. The elusive path of brain tissue oxygenation and cerebral perfusion in harness hang syncope in mountain climbers. High Alt Med Biol 2017; 18: $363-371$

[3] Mortimer RB. Risks and management of prolonged suspension in an Alpine harness. Wilderness Env Med 2011; 22: 77-86

[4] Flora G, Hölzl H. Fatal and non-fatal accidents involving falls into the rope. 2. Bergrettungsärztetagung. Innsbruck, 1972

[5] Adisesh A, Lee C, Porter K. Harness suspension and first aid management: development of an evidence-based guideline. Emerg Med J 2011; 28: 265-268

[6] Kolb JJ, Smith EL. Suspension Shock. Redefining the diagnosis \& treatment of suspension trauma. J Emerg Med Serv 2015; 40: $48-51$

[7] Wharton DR, Mortimer RB. Rhabdomyolysis after prolonged suspension in a cave. Wilderness Env Med 2011; 22: 52-53

[8] Madsen P, Svendsen LB, Jorgensen LG et al. Tolerance to headup tilt and suspension with elevated legs. Aviat Space Env Med 1998; 69: $781-784$

[9] Orzech M, Goodwin M, Brickley J et al. Test program to evaluate human response to prolonged motionless suspension in three types of fall protection harnesses. Harry G Armstrong Aerospace Medical Research Laboratory, Wright-Patterson Air Force Base, OH, 1987. Im Internet: http://www.dtic.mil/dtic/ tr/fulltext/u2 / a262508.pdf Stand: 30.05.2018

[10] Lee C, Porter KM. Suspension trauma. Emerg Med ] 2007; 24: $237-238$

[11] Kinsella SM, Tuckey JP. Perioperative bradycardia and asystole: relationship to vasovagal syncope and the Bezold-Jarisch reflex. $\mathrm{Br}$ J Anaesth 2001; 86: 859-868

[12] Weimer LH, Zadeh P. Neurological Aspects of Syncope and Orthostatic Intolerance. Med Clin North Am 2009; 93: 427 449

[13] Ventzke M. Plötzliche Bewusstlosigkeit - Synkope im Notarztdienst. Der Notarzt 2016; 32: 292-298

[14] Alboni P, Brignole M, Degli Uberti EC. Is vasovagal syncope a disease? Europace 2007; 9: 83-87

[15] Cervellin G, Comelli I, Lippi G. Rhabdomyolysis: historical background, clinical, diagnostic and therapeutic features. Clin Chem Lab Med 2010; 48: 749-756

[16] Allison RC, Bedsole DL. The other medical causes of rhabdomyolysis. Am J Med Sci 2003; 326: 79-88
[17] Bosch X, Poch E, Grau JM. Rhabdomyolysis and Acute Kidney Injury. N Engl J Med 2009; 361: 62-72

[18] Larcher M, Zak H. Seiltechnik. 7. Aufl. Innsbruck: Österreichischer Alpenverein; 2017

[19] Semmel C, Semmel C. Klettern: Sicherung und Ausrüstung. 5. überarbeitete Aufl. München: BLV Buchverlag; 2017

[20] Winkler K, Brehm HP, Haltmeier J. Bergsport Sommer: Technik, Taktik, Sicherheit. SAC-Verlag 2015

[21] Thomassen O, Skaiaa SC, Brattebo G et al. Does the horizontal position increase risk of rescue death following suspension trauma? Emerg Med J 2009; 26: 896-898

[22] Hsiao $\mathrm{H}$, Turner $\mathrm{N}$, Whisler $\mathrm{R}$ et al. Impact of harness fit on suspension tolerance. Hum Factors 2012; 54: 346- 357

[23] Occupational Safety and Health Administration. Suspension Trauma/Orthostatic Intolerance. Safety and Health Information Bulletin (2011). Im Internet: https://www.osha.gov/dts/ shib/shib032404.html Stand: 30.05.2018

[24] Lechner R, Lobensteiner T. Hängetrauma. In: Neitzel C, Ladehof K, Hrsg. Taktische Medizin: Notfallmedizin und Einsatzmedizin. Berlin, Heidelberg: Springer; 2015: 315-321

[25] Glatterer S. Harness Suspension Trauma. MERIDIAN Q Publ Mt Rescue Assoc 2011. Im Internet: http://mra.org/wp-content/ uploads/2016/05/meridianapr2011.pdf Stand: 06.06.2018

[26] Stuhlinger W, Dittrich P, Flora G et al. Circulatory and renal function changes in test subjects suspended from the upper half of the body. 2. Bergrettungsärztetagung. Innsbruck, 1972

[27] Soar J, Nolan JP, Böttiger BW et al. Adult advanced life support section Collaborators. European Resuscitation Council Guidelines for Resuscitation 2015: Section 3. Adult advanced life support. Resuscitation 2015; 95: $100-147$

[28] Reinertson R. Suspension trauma and rhabdomyolysis. Wilderness Environ Med 2011; 22: 286 - 287 author reply 287288

[29] Truhláŕ A, Deakin CD, Soar J et al. Cardiac arrest in special circumstances section Collaborators. European Resuscitation Council Guidelines for Resuscitation 2015: Section 4. Cardiac arrest in special circumstances. Resuscitation 2015; 95: 148 201

\section{Bibliografie}

DOI https://doi.org/10.1055/a-0796-0151

retten 2019; 8: 112-120

(c) Georg Thieme Verlag KG, Stuttgart · New York

ISSN 2193-2387 


\section{Punkte sammeln auf CMEthieme.de}

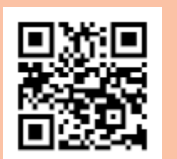

Diese Fortbildungseinheit ist in der Regel 12 Monate online für die Teilnahme verfügbar.

Den genauen Einsendeschluss finden Sie unter https://eref.thieme.de/CXB4WIQ.

Sollten Sie Fragen zur Online-Teilnahme haben, finden Sie unter https://cme.thieme.de/hilfe

eine ausführliche Anleitung. Wir wünschen viel Erfolg beim Beantworten

der Fragen!

Unter https://eref.thieme.de/CXB4WIQ oder über den QR-Code kommen Sie

direkt zur Startseite des Wissenstests.

\section{Frage 1}

Wodurch ist das Hängetrauma gekennzeichnet?

A Äußere Verletzungen sind die Regel.

B Es handelt sich um ein ungesichertes Sturzereignis.

C Kopfüber hängender Betroffener.

D Es handelt sich ausschließlich um Unfälle in alpinem Gelände.

E Bewegungsloses Hängen kann ein Hängetrauma auslösen.

\section{Frage 2}

Wie wirkt sich das Hängetrauma auf das Herz-Kreislauf-System nicht aus?

A kardiale Arrhythmie

B abgeflachte T-Welle aufgrund einer Hyperkaliämie

$C$ reduzierte kardiale Vorlast

D reduziertes Herzminutenvolumen

E venöses Blutpooling

\section{Frage 3}

Wie gefährlich ist das Hängetrauma?

A immer lebensgefährlich

B nur bei bestehender Komorbidität manchmal lebensbedrohlich

C nur für ältere Personen ab 60 Jahre gefährlich

D potenziell lebensbedrohlich

E ungefährlich

\section{Frage 4}

Nach welcher Hängezeit können die ersten Symptome eines Hängetraumas auftreten?
A sofort
$B$ einige Minuten
$C$ eine halbe Stunde
D eine Stunde
E zwei Stunden

\section{Frage 5}

Eine der folgenden Aussagen zur Auswirkung eines Hängetraumas auf die Niere ist falsch. Welche?

A Bei Hängezeiten von über 2 Stunden sollte eine Zielklinik mit der Möglichkeit zu Nierenersatzverfahren angefahren werden.

B Die Minderperfusion der Nieren kann bei langen Hängezeiten eine akute Nierenschädigung verursachen.

C Eine Crush-Niere ist mögliche Folge eines Hängetraumas.

D Im Gegensatz zum prärenalen ist ein intrarenales Nierenversagen durch ein Hängetrauma nicht auszulösen.

E Unter anderem die mit der Rhabdomyolyse verbundene Myoglobinämie kann bei langen Hängezeiten eine akute Nierenschädigung auslösen.

\section{Frage 6}

Wodurch ist Prävention eines Hängetraumas charakterisiert?

A Eine Prävention ist nicht möglich.

B Gefährdete Personen sollten Selbstrettungstechniken beherrschen.

C Die Hängezeit hat keinen Einfluss auf die Prävention des Hängetraumas.

D Es gibt keine Materialien/Ausrüstungsgegenstände, die in der Prävention des Hängetraumas hilfreich wären.

E Betroffene sollten immer auf die professionellen Helfer warten.

\section{Frage 7}

Kameradenhilfe bei Hängetrauma ...

A ist gewöhnlich nicht verfügbar, da Alpinisten als Individualsportler am liebsten allein unterwegs sind.

$B$ ist weit weniger bedeutsam für die Prognose als die professionelle Rettungskette.

C kann vernachlässigt werden.

D sollte zum Ziel haben, die hängende Person so schnell wie möglich aus dieser Situation zu befreien.

E verschlimmert die Situation in der Regel nur.

- Weitere Fragen auf der folgenden Seite... 


\section{Punkte sammeln auf CME.thieme.de}

\section{Frage 8}

Eine der folgenden Aussagen zu Selbstrettungsmaßnahmen ist falsch. Welche?

A Die Beine können durch Anbringen einer möglichst breiten Schlinge unterhalb der Knie in eine annähernd horizontale Position gebracht werden.

B Empfohlen werden auch Trittschlingen, die mithilfe von Klemmknoten oder Klemmgeräten am Seil befestigt werden können, um durch wiederholtes Hineintreten die Muskelpumpe und damit den venösen Blutfluss zu aktivieren.

C Es kann die Muskelpumpe aktiviert werden.

D Forcierte Bewegung der Beine (z. B. „Radfahren“, Abdrücken gegen ein Widerlager) sollten erfolgen.

E Da die meisten Betroffenen bewusstlos sind, erübrigt sich die Selbstrettung.

\section{Frage 9}

Wie sollten Anseilgurte unter dem Aspekt der Prävention von Hängetraumata idealerweise beschaffen sein?

A Es sollten möglichst Brustgurte ohne Sitzgurt verwendet werden.

B Das direkte Einbinden mit dem Seil um den Körper ermöglicht im Zweifelsfall eine schnelle Rettung.

C Der Anseilpunkt sollte am Rücken liegen.

D Günstig ist ein Einbindepunkt möglichst hoch sternal.

E Der Gurt ist so beschaffen, dass sich beim freien Hängen eine halb horizontale Position einstellt.

\section{Frage 10}

Eine der folgenden Aussagen zum rettungsdienstlichen Vorgehen bei Hängetrauma ist falsch. Welche?

A Bei bewusstlosen Patienten ist es wichtig, durch Überstrecken des Kopfes für einen freien Atemweg zu sorgen.

$B$ Eigenschutz muss unbedingt beachtet werden und genießt oberste Priorität.

C Es wurde durch mehrere Studien nachgewiesen, dass der sog. Bergungstod durch eine akute Volumenüberlastung des Herzens eintritt, wenn der Patient sofort nach der Bergung initial flach gelagert wird.

D So bald wie möglich sollte die Standardbehandlung nach dem cABCDE-Algorithmus erfolgen.

E Zur Prävention eines Nierenversagens im Rahmen einer wahrscheinlichen Rhabdomyolyse ist bei langen Hängezeiten eine liberale kristalloide Flüssigkeitsgabe indiziert. 